\title{
TRANSFORMAÇÃO DE HELMERT GENERALIZADA NO POSICIONA- MENTO DE ALTA PRECISÃO: FUNDAMENTAÇÃO TEÓRICA E EXEMPLIFICAÇÕES
}

\author{
L. F. Sapucci ${ }^{1} \&$ J. F. G. Monico ${ }^{2}$
}

Received March 23, 2000 / Accepted June 26, 2001

\begin{abstract}
A implantação de um referencial que descreve posições na superfície terrestre, ou próximo a essa, é dividida em duas fases: definição do sistema de referência (Reference System) e sua materialização (Reference Frame). As técnicas de posicionamento que empregam satélites artificiais, como o GPS (Global Positioning System) e o GLONASS (Global Navigation Satellite System), necessitam utilizar referenciais que materializam sistemas de referência bem definidos. Tais técnicas têm sido utilizadas com freqüência em atividades de geodinâmica, pois atendem a exigência quanto a alta precisão dos resultados, essencial nessas aplicações. Para essas técnicas estão disponíveis as realizações do WGS84 (World Geodetic System 1984), do PZ90 (Parametry Zemli 1990) e as várias do ITRS (IERS -International Earth Rotation Service- Terrestrial Reference System). Nestas atividades, freqüentemente, necessita-se comparar soluções materializadas em diferentes referenciais, as quais podem estar associados a diferentes épocas. Para tanto, deve-se empregar a transformação de Helmert generalizada, a qual é apropriada para aplicações tetradimensionais. Neste artigo apresentam-se as características relevantes de tal transformação, os parâmetros de transformação disponíveis e as possíveis versões que ela pode assumir, exemplificando-as com aplicações.
\end{abstract}

Palavras-chave: Transformação de Helmert Generalizada; Vetor Velocidade; Parâmetros de Transformação; Geodésia 4D.

GENERALIZED HELMERT TRANSFORMATION FOR HIGH PRECISION POSITIONING: THEORY AND EXAMPLES - The implementation of a referential that describes positions in the terrestrial surface, or near it, is divided in two phases: the definition (Reference System) and the materialization (Reference Frame). The positioning techniques that use artificial satellites, like GPS (Global Positioning System) and GLONASS (Global Navigation Satellite System), need to use reference frames, which materialize well-defined reference systems. These techniques have been used frequently in geodynamics activities, because of the high precision provided, which is essential to these applications. For such techniques are available the realizations of WGS84 (World Geodetic System 1984), of PZ90 (Parametry Zemli 1990) and of the several ITRS (IERS-International Earth Rotation Service- Terrestrial Reference System). In these activities it is frequently needed to compare solutions materialized in different frames, which can be associated to different epochs. For that, it should be used the generalized Helmert transformation which is appropriate for $4 D$ applications. This article presents the most relevant characteristics of this transformation, the available transformation parameters and the possible versions that it can assume, exemplifying them with applications.

Key words: Generalized Helmert Transformation; Velocity field; Transformation Parameters; 4D Geodesy.

\footnotetext{
${ }^{1}$ Faculdade de Ciências e Tecnologia - UNESP

${ }^{2}$ Departamento de Cartografia, FCT/UNESP

Rua Roberto Simonsen, 305, CEP 19060-900, Cx. Postal: 957

Presidente Prudente, SP, Brasil, fone: (18) 229-5325

'sapucci@prudente.unesp.br ${ }^{2}$ galera@prudente.unesp.br
} 


\section{INTRODUÇÃO}

Para a determinação de uma posição sobre a superfície terrestre necessita-se utilizar um sistema de coordenadas. Sua construção abrange as etapas de concepção, definição, realização e densificação. As duas primeiras são denominadas como sistema de referência (Reference System) e as duas restantes como rede de referência (Reference Frame) (Oliveira, 1998). O sistema de referência, existente no espaço abstrato, é pertinente aos princípios conceituais que objetivam o sistema de coordenadas. A rede de referência, por sua vez, particulariza um conjunto de estações que possibilitam a descrição dos valores da posição e movimento de objetos relativos ao sistema de referência. Na literatura brasileira as duas primeiras etapas também são denominadas por referencial definido e as duas últimas por referencial materializado (Gemael, 1994). Essa última denominação é a que será utilizada nesse trabalho.

Para as aplicações do posicionamento geodésico que envolvem a utilização de satélites artificiais, tais como o GPS (Global Positioning System) e o GLONASS (Global Navigation Satellite System), há diversos referenciais materializados disponíveis. O referencial associado aos resultados finais obtidos por essas técnicas é forçosamente o mesmo empregado para referenciar as efemérides dos satélites utilizados no processamento dos dados. As efemérides preditas do GPS estão referenciadas ao WGS84 (World Geodetic System 1984) e no caso do GLONASS ao PZ90 (Parametry Zemli 1990). As efemérides precisas, produzidas pelo IGS (International GPS Service), tanto do GLONASS como do GPS, estão referenciadas ao ITRF (IERS -International Earth Rotation Service- Terrestrial Reference Frame). Por isso, para um mesmo ponto, pode-se obter valores distintos para suas coordenadas, dependendo do referencial adotado.

As aplicações do GPS e GLONASS em geodinâmica contribuem para os estudos das mudanças globais ocorridas no planeta. Elas possibilitam o entendimento de fenômenos geofísicos que ocorrem ao longo do tempo, como por exemplo o movimento das placas litosféricas e a avaliação de abalos sísmicos. Para tal, necessita-se efetuar medições com alta precisão, possibilitando a determinação das coordenadas de estações terrestres com nível de acurácia adequado à geodinâmica. Atualmente, há algumas técnicas que podem atingir acurácia de poucos ppb (partes por bilhões), as quais são classificadas como de alta precisão. O GPS é um dessas técnicas. Cuidados especiais devem ser tomados para que as coordenadas das estações terrestres, consideradas conhecidas, estejam no mesmo referencial das efemérides utilizadas (WGS84, PZ90 ou ITRF), assim como a compatibilidade em termos de época. A variável tempo é importante devido as alterações nas coordenadas, provocadas pela continua deformação da crosta terrestre. Para compatibilizar diferentes referenciais, considerando a evolução temporal das coordenadas, necessita-se de uma transformação que aplica simultaneamente as translações, rotações, fator de escala e respectivas taxas de variação com relação ao tempo, além da velocidade da estação. Ela é denominada transformação de Helmert generalizada (Gregorius, 1996), que é uma forma geral da transformação de Helmert.

De forma geral, a utilização da transformação de Helmert generalizada é necessária em aplicações de alta precisão. Caso contrário, a incerteza das coordenadas resultantes seria maior que as variações devido a inconsistência dos referenciais envolvidos. Conseqüentemente, as contribuições ao utilizar esse tipo de transformação seriam imperceptíveis.

A transformação de Helmert generalizada, assim batizada por Gregorius (1996), tem sido também denominada por transformação rigorosa entre diferentes referenciais (Soler, 1998; 1999). No entanto, esses autores apresentaram aplicações dessa transformação sem se reportar aos seus fundamentos básicos. Esse artigo objetiva apresentar os conceitos fundamentais dessa transformação, sua dedução analítica e as diferentes versões que pode assumir, além de apresentar como utilizá-la.

Para alcançar tais objetivos é apresentada a teoria básica em que está inserida a transformação de Helmert generalizada, alguns aspectos relacionados aos referencias mais utilizados pelas técnicas espaciais de posicionamento geodésicos (WGS84, PZ90 e as séries do ITRF) e os parâmetros de transformação disponíveis entre esses referenciais. Será feito um relato referente ao movimento das placas litosféricas e por fim, visando alcançar os objetivos propostos, serão apresentadas as diferentes versões desta transformação, exemplificando-as e discutindo seus resultados.

\section{FUNDAMENTAÇÃO TEÓRICA DA TRANS- FORMAÇÃO DE HELMERT GENERA- LIZADA}

Tomando as coordenadas de um ponto $P$ qualquer, 
associadas a um referencial $A$, em uma época de referência $\left(t_{o}\right)$, pode-se obter suas coordenadas no referencial $B$ em uma outra época $(t)$, com a aplicação da transformação de Helmert generalizada, da seguinte forma (Soler, 1999):

$$
\begin{gathered}
\vec{X}_{B(t)}=\vec{T}+(1+s)[\varepsilon+I]\left[\vec{X}_{A\left(t_{0}\right)}+\vec{V}_{A\left(t_{0}\right)}\left(t-t_{0}\right)\right]+ \\
{\left[\dot{\vec{T}}+[(1+s) \dot{\varepsilon}+\dot{s}[\varepsilon+I]] \vec{X}_{A\left(t_{0}\right)}\right]\left(t-t_{0}\right)}
\end{gathered}
$$

onde :

$\vec{T}$ é a translação necessária para que coincida as origens dos referenciais $A$ e $B$, em outras palavras, é as coordenadas da origem de $A$ no referencial $B$, na época de referencia $t_{0}$;

$\varepsilon$ é a matriz de rotações diferenciais, em radianos, em torno dos eixos $X, Y, Z$ do referencial $A$ para estabelecer paralelismo com $B$, na época $t$;

$s$ é o fator diferencial de escala entre os referenciais $A$ e $B$ na época $t_{0}$;

$\vec{X}_{A\left(t_{0}\right)}$ é o vetor das coordenadas cartesianas do ponto $P$ no referencial $A$, na época de referencia $t_{o}$ em unidades métricas;

$\vec{X}_{B(t)}$ é o vetor das coordenadas cartesianas do ponto $P$ no referencial $B$, na época $t$ em unidades métricas;

$$
\vec{V}_{A\left(t_{0}\right)} \text { é o vetor velocidade do ponto } P \text { (em }
$$
unidades métricas por ano), na época $t_{0}$, devido ao movimento da placa litosférica que o contém;

$\left(t-t_{o}\right)$ é o intervalo de tempo expresso em anos e sua fração. $\mathrm{O}$ valor de $t$ na prática é aproximado para o tempo médio do período observado; e

$\dot{\vec{T}}, \dot{\varepsilon}, \dot{s}$ são as variações em translação, rotação e fator diferencial de escala, respectivamente, entre os referenciais $A$ e $B$, com relação ao tempo.

Para uma melhor compreensão da equação apresentada acima, será feita a seguir sua dedução analítica. Tomando um ponto $P$ em um referencial $A$, a transformação de Helmert desta coordenada para um outro referencial $B$ (ambas materializações de sistemas dextrogiros), pode ser escrita como (Bock, 1998):

$\vec{X}_{B}=\vec{T}+(1+s) R \vec{X}_{A}$,

onde $\vec{X}_{A}$ e $\vec{X}_{B}$ são as coordenadas do ponto $P$ nos respectivos referenciais; $\vec{T}$ é o vetor das coordenadas da origem do referencial $A$ em relação ao $B ; s$ é o fator diferencial de escala e $R$ é a matriz ortogonal de rotação do referencial $A$ para $B$.

Como a Terra é um corpo não-rígido, as coordenadas do ponto $P$ alteram-se com a taxa de variação $\vec{V}_{A\left(t_{0}\right)}$, referenciada em $A$, que é determinada a partir da variação média de sua posição com relação ao tempo. Assim, para um intervalo de tempo entre $t_{0}$ e $t$ tem-se:

$$
\begin{gathered}
\vec{V}_{A\left(t_{0}\right)}=\frac{\vec{X}_{A(t)}-\vec{X}_{A\left(t_{0}\right)}}{\left(t-t_{0}\right)} ; \\
\vec{X}_{A(t)}=\vec{X}_{A\left(t_{0}\right)}+\vec{V}_{A\left(t_{o}\right)}\left(t-t_{0}\right) .
\end{gathered}
$$

Da mesma forma, as coordenadas deste ponto no referencial $B\left(\vec{X}_{B(t)}\right)$, em um instante $t$, são determinadas por :

$$
\vec{X}_{B(t)}=\vec{X}_{B\left(t_{0}\right)}+\vec{V}_{B\left(t_{o}\right)}\left(t-t_{0}\right)
$$

A Eq.(2) pode ser escrita na época inicial $t_{o}$ na seguinte forma:

$$
\vec{X}_{B\left(t_{0}\right)}=\vec{T}+(1+s) R \vec{X}_{A\left(t_{0}\right)},
$$

cuja derivada é:

$$
\begin{aligned}
\vec{V}_{B\left(t_{0}\right)}= & \dot{\vec{T}}+[(1+s) \dot{R}+\dot{s} R] \vec{X}_{A\left(t_{0}\right)}+ \\
& +(1+s) R \vec{V}_{A\left(t_{0}\right)}
\end{aligned}
$$

Substituindo (6) e (7) na Eq.(5) tem-se:

$$
\begin{aligned}
\vec{X}_{B(t)}= & \vec{T}+(1+s) R \vec{X}_{A(t o)}+\left[\dot{\vec{T}}+[(1+s) \dot{R}+\dot{s} R] \vec{X}_{A\left(t_{0}\right)}+\right. \\
& \left.+(1+s) R \vec{V}_{A\left(t_{0}\right)}\right]\left(t-t_{0}\right) .
\end{aligned}
$$

Associando os termos em comum, tem-se:

$$
\begin{aligned}
\vec{X}_{B(t)} & =\vec{T}+(1+s) R\left[\vec{X}_{A(t o)}+\vec{V}_{A(t o)}\left(t-t_{0}\right)\right]+ \\
& +\left[\dot{\vec{T}}+[(1+s) \dot{R}+\dot{s} R] \vec{X}_{A(t o)}\right]\left(t-t_{o}\right)
\end{aligned}
$$

Para rotações pequenas, a matriz de rotação $R$ pode ser reduzida para

onde

$$
R=\varepsilon+I,
$$




$$
\varepsilon=\left[\begin{array}{ccc}
0 & e_{Z} & -e_{Y} \\
-e_{Z} & 0 & e_{X} \\
e_{Y} & -e_{X} & 0
\end{array}\right]
$$

Substituindo a Eq.(10) na Eq.(9), chega-se a Eq.(1) como se desejava.

$\mathrm{Na}$ equação da transformação de Helmert generalizada (Eq.(1)) pode-se notar a presença do termo

$$
(1+s)[\varepsilon+I],
$$

que pode ser expandido para:

$$
\varepsilon+I+s \varepsilon+s I \text {. }
$$

Em muitas aplicações a Eq.(13) pode ser simplificada por

$$
\varepsilon+(1+s) I=\left[\begin{array}{ccc}
(1+s) & e_{Z} & -e_{Y} \\
-e_{Z} & (1+s) & e_{X} \\
e_{Y} & -e_{X} & (1+s)
\end{array}\right]
$$

Isto é devido ao fato da matriz de rotação e o fator diferencial de escala serem constituidos de valores pequenos, de forma que a contribuição do produto desses termos é insignificante para muitas aplicações, como aquelas que utilizam uma simples transformação de Helmert. Isto não ocorre na transformação de Helmert generalizada, onde sua precisão é sensível a estas modificações e devem ser evitadas, aplicando todos os termos, como é apresentado na Eq.(1).

Ta como as coordenadas, a velocidade de um ponto $P$ associado ao referencial $B$ também pode ser obtida a partir de uma velocidade conhecida no referencial $A$, utilizando-se da seguinte fórmula:

$$
\begin{aligned}
\vec{V}_{B}= & \dot{\vec{T}}+[(1+s) \dot{\varepsilon}+\dot{s}[\varepsilon+I]] \vec{X}_{A}+ \\
& +(1+s)[\varepsilon+I] \vec{V}_{A}
\end{aligned}
$$

onde $\vec{V}_{B}$ representa as componentes lineares referidas aos eixos cartesianos do campo de velocidade associado ao ponto $P$, no referencial $B$. Os termos restantes contidos na Eq.(15) são análogos aos da Eq.(1).

A demonstração da Eq.(15) pode ser obtida partindo do fato de que as coordenadas de um ponto em dois referenciais diferentes, $A$ e $B$, estão relacionadas pela Eq.(6). A variação das coordenadas desse ponto no referencial $B$ com relação ao tempo, é dada em função da velocidade com que o mesmo ponto se move em $A$, ou seja, pela Eq.(7). Assim, substituindo a Eq.(10) na Eq.(7), chega-se na Eq.(15), que é o resultado desejado. O valor de $\dot{\vec{T}}$, em geral é negligenciado, tal como consta em Soler $(1998 ; 1999)$. Isto se deve a imprecisão em sua determinação.

A Eq.(15) pode ser aplicada nos casos em que se conhece a velocidade da estação em um determinado referencial, e deseja-se utilizá-la em um outro, cujo campo de velocidade não é conhecido. Porém, para aplicar a Eq.(15) é necessário conhecer os parâmetros de transformação e suas taxas de variação com relação ao tempo, entre os referenciais envolvidos, que nem sempre estão disponíveis. Quando isso ocorre, devese utilizar outros recursos que serão apresentados posteriormente nesse trabalho.

Os referenciais contidos nas equações anteriores denominados por $A$ e $B$, podem assumir as diversas materializações do ITRS, WGS84, PZ90, ou qualquer outro referencial apropriado.

\section{REFERENCIAIS TERRESTRES CONVEN- CIONAIS}

Atualmente são grandes as vantagens da utilização de satélites no posicionamento geodésico (GPS ou GLONASS). Essas vantagens tornaram-se evidentes desde os primeiros testes, provando ser técnicas bastante eficientes. Ao utilizá-los, as coordenadas finais podem ser expressas em três possíveis referenciais, dos quais serão apresentados a seguir seus aspectos mais relevantes. Trata-se das materializações do WGS84, do PZ90 e as várias do ITRS (denominadas ITRF):

- WGS84 (World Geodetic System 1984): é o referencial desenvolvido pela agência norte-americana National Imagery and Mapping Agency (NIMA) ligada ao Departamento de Defesa dos EUA (DoD). Sua origem é o centro de massa da terra e os eixos $X$, $Y$ e $Z$ idênticos ao do Sistema Convencional Terrestre (CTS) tal como definido pelo BIH (Bureau International de L'Heure) para a época de 1984,0. O elipsóide utilizado coincide com o do GRS80 (Geodetic Reference System 1980). A sua materialização é a utilizada pelo GPS para referenciar as efemérides transmitidas. As coordenadas provenientes do processamento de dados utilizando essas efemérides, estão associadas e esse referencial. Apesar de sua realização inicial pertencer a era Doppler, atualmente está quase que exclusivamente baseado em observações GPS, com freqüentes refinamentos para 
melhorar sua precisão. A solução mais recente é o denominado WGS84 (G873) que passou a ser utilizado pelo NIMA desde 29 de janeiro de 1997 para referenciar as efemérides transmitidas (Malys et al., 1997).

- PZ90 (Parametry Zemli 1990): é formalmente conhecido por Sistema Geodésico Soviético 1990 (Soviet Geodetic System 1990). Tem definição similar à do ITRF, com a origem no centro de massa da terra. O eixo $Z$ é direcionado para o Pólo Norte Médio da época 1900-1905, o eixo $X$ está no plano do equador também da época 1900-1905 com o plano XZ sendo paralelo ao Meridiano Médio de Greenwich, formando um sistema dextrógiro. Suas maiores deficiências em sua materialização estão no baixo número de estações de controle e na sua má distribuição global, além da precária manutenção. Esse referencial não sofreu modificações significativas desde 1993 o que tem gerado efemérides pouco confiáveis.

- ITRF (IERS - International Earth Rotation Service - Terrestrial Reference Frame): é a realização do ITRS, o qual é um sistema de referência convencional terrestre (CTRS) definido por uma série de modelos e definições (McCarthy, 1992; 1996). Foi estabelecido e é mantido pelo IERS (International Earth Rotation Service) com sede em Paris. A obtenção do ITRF resulta da combinação de uma lista de coordenadas (com seus valores de variância e covariância) e de velocidades de estações (SSCs - Set of Station Coordinates), derivadas de observações de várias técnicas geodésicas espaciais, tais como VLBI (Very Long Baseline Interferometry), LLR (Lunar Laser Ranging), SLR (Satellite Laser Ranging) e o GPS nas soluções mais recentes (desde o ITRF91) (Monico \& Segantine, 1996). A primeira solução ITRF publicada foi o ITRF88, e sucessivas versões tem sido publicadas, até a mais atual, denominada ITRF2000. Quando as coordenadas forem expressas em latitude $(\varphi)$, longitude $(\lambda)$ e altitude $(h)$ o elipsóide a adotar é o GRS80, recomendado pela IUGG (International Union of Geodesy and Geophysics). O ITRF é utilizado pelos centros de análises do IGS para referenciar as efemérides precisas do GPS, assim como as efemérides precisas dos satélites GLONASS, resultante do IGEX98 (International GLONASS Experiment 1998). Tais produtos são disponibilizados na Internet por diversas instituições via ftpanomymous. Uma destas instituições é a NASA (National Aeronautics and Space Administration) que disponibiliza efemérides precisas para o GPS e para o GLONASS, além de outros produtos, pelo seguinte
Tabela 1-Referenciais utilizados na geração das efemérides do IGS.

Table 1-Reference frame used in the generation of the IGS ephemerides.

\begin{tabular}{|cc|c|}
\hline \multicolumn{2}{|c|}{ P eríodo da efeméride } & \multirow{2}{*}{$\begin{array}{c}\text { ITRF } \\
\text { Utilizado }\end{array}$} \\
\hline \hline Início & Fim & ITRF91 \\
Jan. 1993 & Dez. 1993 & ITRF92 \\
Jan. 1994 & Dez. 1994 & ITRF93 \\
Jan. 1995 & Jun 1996 & ITRF94 \\
Jul. 1996 & Fev. 1998 & ITRF96 \\
Mar.1998 & Jul. 1999 & ITRF97 \\
Ago. 1999 & Dias atuais \\
\hline
\end{tabular}

endereço $<$ ftp cddisa.gsfc.nasa.gov $>$.

As efemérides precisas dos satélites GPS, produzidas pelo IGS, foram desde o inicio, baseadas nas realizações do ITRF, como mostra a Tab.(1). Da mesma forma, as primeiras efemérides precisas produzidas para os satélites GLONASS estão também referenciadas ao ITRF, neste caso para o ITRF94.

Para a aplicação da transformação de Helmert generalizada é necessário que se conheça o vetor velocidade com que as coordenadas das estações são alteradas com o decorrer do tempo, devido às deformações da crosta terrestres. No caso do ITRF as velocidades das estações são fornecidas juntamente com suas coordenadas. Para outros referenciais que não possuem um campo de velocidades nele associado, como é o caso do WGS84 e do PZ90, valores aproximados para essas velocidades podem ser obtidos ao aplicar modelos geofísicos de movimento de placas litosféricas.

\section{MOVIMENTO DAS PLACAS LITOSFÉ- RICAS}

A determinação do movimento das placas que compõem a parte superior da Terra justifica o rigor com que deve ser tratada uma transformação entre diferentes referenciais de alta precisão. Os movimentos das placas geram alterações nas coordenadas das estações com o passar do tempo, de tal forma que uma simples transformação de Helmert não é suficiente, sendo necessário considerar as variações dos parâmetros envolvidos nessa transformação.

Para prever este movimento foram desenvolvidos vários modelos baseados em informações geofísicas e geológicas de milhões de anos. Entre eles pode-se destacar o AMO (Absolute Plate Motion Model), o AKLM8.8 no qual também considera observações geodésicas espaciais (VLBI, SLR e GPS) e o mais 
conhecido, o qual é recomendado pelo IUGG (McCarthy, 1996), o NNR-NUVEL-1A (No Net Rotation - Northern University Velocity Model 1A). A confiabilidade do modelo NNR-NUVEL-1A é sustentada pelo fato do mesmo combinar várias informações, tais como variações de anomalias magnéticas, azimutes de falhas na crosta, vetores de terremotos e a separação da crosta oceânica para estimar a velocidade relativa de cada placa litosférica (Costa, 1999). O NNR-NUVEL-1A descreve as velocidades angulares das placas litosféricas que compõem a crosta terrestre, tomando como referência a placa do Pacífico e definidas sobre a condição de que é uma rede sem rotações residuais (No Net Rotation). Essa injunção faz com que a resultante das deformações das placas seja nula (Bock, 1998).

O movimento relativo das placas litosféricas resultante destes modelos é descrito pelos vetores de rotação de Euler que são proporcionais as velocidades angulares, podendo por isso ser transformados em velocidades nas estações. Em McCarthy (1996) são apresentadas as componentes de velocidade angular resultante do modelo geofísico NNR-NUVEL-1A para cada uma das placas litosféricas que compõem a superfície terrestre.

Na Tab.(2) são apresentados os valores de $\Omega x$, $\Omega y$ e $\Omega z$ da placa litosférica América do Sul, segundo os modelos NNR-NUVEL-1A, APKIM8.8 e os valores calculados para o Brasil, obtidos a partir dos resultados fornecidos pelas 10 estações da RBMC (Rede Brasileira de Monitoramento Contínuo) (Costa, 1999). Tais valores são expressos em segundos por milhões de anos, pois é mais intuitivo visualizar uma rotação quando se utiliza essa unidade.

Desde as primeiras realizações do ITRS os movimentos das placas litosféricas foram considerados e a consistência de seus resultados foi analisada ao comparar com os modelos geofísicos descritos anteriormente (Boucher \& Altamini, 1996). Do ITRF88 até o ITRF90, o modelo de movimento das placas

Tabela 2 - Vetores de rotação da Placa América do Sul segundo os modelos: NNR-NUVEL-1A, APKIM8.8 e os resultantes da RBMC (Costa, 1999).

Table 2 - Rotation vectors of the South American plate based on NNR-NUVEL-1A, APKIM8.8 and results of RBMC (Costa, 1999).

\begin{tabular}{|c|c|c|c|}
\hline Modelo & $\Omega_{x \text { (segM.ano) }}$ & $\Omega_{y \text { (seg/M.ano) }}$ & $\Omega_{Z \text { (seg/M.ano) }}$ \\
\hline NNR-NUV EL-1A & $-214,10$ & $-312,49$ & $-179,45$ \\
APKIM8.8 & $-417,96$ & $-192,96$ & $-144,36$ \\
RBMC & $-578,52$ & $-344,52$ & $-223,56$ \\
\hline
\end{tabular}

litosféricas AMO-2 foi utilizado para associar um modelo de velocidades em suas realizações. Desde o ITRF91, os campos de velocidades globais foram ajustados combinando velocidades derivadas de VLBI, SLR e do GPS (este último desde 1993). Constatou-se que a evolução temporal do ITRF91 e do ITRF92 são consistentes com o modelo geofísico NNR-NUVEL1. Quanto ao ITRF93, sua evolução temporal foi forçada para ser consistente com os parâmetros de orientação da Terra (EOP-Earth Orientation Parameters), do IERS, originando com relação ao modelo geofísico mais recente, NNR-NUVEL-1A, variações dos parâmetros de transformação, as quais serão apresentadas posteriormente. A evolução temporal do ITRF94 é consistente com o modelo NNRNUVEL-1A (Boucher \& Altamini, 1996), assim como as demais realizações que o sucederam. No que concerne ao WGS84, para prever a evolução temporal das estações pertencentes aos refinamentos mais recentes foi tratada com o modelo NNR-NUVEL-1A.

Muitas vezes não se conhece a velocidade com que as coordenadas de uma determinada estação terrestre varia com relação ao tempo. Essa é uma informação importante, principalmente quando se quer avaliar a compatibilidade entre as coordenadas de uma estação associadas em diferentes referenciais, obtidos em épocas distintas. Valores aproximados do vetor velocidade nessas estações podem ser determinados utilizando as velocidades angulares provenientes de modelos geofísicos, como por exemplo o NNRNUVEL-1A. Isto é obtido pela seguinte expressão (Soler,1998):

$$
\vec{V}_{A} \approx \Omega_{P i} \vec{X}_{A}
$$

onde $\vec{V}_{A}$ é o vetor velocidade e $\vec{X}_{A}$ o vetor das coordenadas cartesianas de uma estação terrestre, ambos referenciados em um referencial qualquer $A \mathrm{e}$ $\Omega_{P i}$ é uma matriz antissimétrica dada por :

$$
\Omega_{P i}=\left[\begin{array}{ccc}
0 & -\Omega_{z} & \Omega_{y} \\
\Omega_{z} & 0 & -\Omega_{x} \\
-\Omega_{y} & \Omega_{x} & 0
\end{array}\right]
$$

onde $\Omega x, \Omega y$ e $\Omega z$ são os valores das velocidades angulares com relação aos eixos $X, Y$ e $\mathrm{Z}$ respectivamente. Esses valores, provenientes do modelo NNR-NUVEL-1A, são apresentados por McCarthy (1996), referente a cada placa litosférica $P i$ e devem ser aplicados na Eq.(16) em rad/ano. Um exemplo da determinação do vetor velocidade de uma 
estação, através da utilização da Eq.(16), é apresentado no Exemplo (1) do Apêndice.

Os valores aproximados do vetor velocidade fornecidos pelo modelo NNR-NUVEL-1A apresentaram compatibilidade satisfatória com os valores obtidos através do ajustamento das estações da RBMC e do IGS, localizadas sobre a placa litosférica América do Sul (Costa,1999). Isto garante a validade deste modelo ao determinar o vetor velocidade aproximada de estações localizadas no território brasileiro para aplicações GPS.

\section{PARÂMETROS DE TRANSFORMAÇÃO}

A aplicação da transformação de Helmert generalizada, como mostra a Eq.(1) envolve a utilização da velocidade da estação e os parâmetros de transformação (com suas respectivas taxas de variação), entre os referenciais desejados. A Tab.(3) apresenta os parâmetros de transformação entre os referenciais mais utilizados no posicionamento geodésico através de satélites artificiais (Malys et al., 1997; McCarthy, 1996; Bazlov et al.,1999).

O ITRF96 e o ITRF97 não estão presentes na Tab.(3), pois entre as materializações mais recentes do ITRF não há parâmetros de transformação, podendo essas, juntamente com o ITRF94, ser consideradas realizações idênticas. Os parâmetros de transformação entre WGS84 (G873) e o ITRF94 foram obtidos através da comparação das coordenadas de 17 estações baseadas nas observações GPS (Malys et al. 1997). Quanto aos parâmetros entre as versões do ITRF, eles são de responsabilidade do IERS (McCarthy, 1996) e foram obtidas por ajustamento de observações provenientes das várias técnicas espaciais envolvidas em sua realização. Por outro lado, os parâmetros entre o PZ-90 e o WGS98 (G873) foram determinados comparando coordenadas absolutas em oito pontos comuns aos dois sistemas (Bazlov et al., 1999).

\section{VERSÕES DA TRANSFORMAÇÃO DE HELMERT GENERALIZADA}

Devido a ausência de parâmetros de transformação entre alguns referenciais (ver Tab.(3)), a transformação de Helmert generalizada pode assumir diferentes versões, que serão apresentadas e exemplificadas a seguir. Para que seja possível exemplificá-las serão utilizadas algumas das mais recentes materializações do ITRS: o ITRF92, ITRF93, ITRF94 e o ITRF96. Como não há nenhuma estação brasileira pertencente ao IRTF92, serão utilizadas as coordenadas da estação ONSA, localizada em Onsala na Suécia. Tais coordenadas são apresentadas na Tab.(4) e foram obtidas através do endereço eletrônico do IERS <http://hpiers.obspm.fr $>$.

As principais versões da transformação de Helmert generalizada são:

- Transformação com 14 parâmetros: é

Tabela 3 - Parâmetros de transformação entre os referenciais mais utilizados no posicionamento geodésico por satélites Table 3 - Transformation parameters between the reference frames most used in the geodetic positioning by satellite.

\begin{tabular}{|c|c|c|c|c|c|c|c|}
\hline $\begin{array}{l}\text { Relação } \\
\text { Funcional }\end{array}$ & $\begin{array}{c}T x \\
(\mathrm{~cm})\end{array}$ & $\begin{array}{c}T y \\
(\mathrm{~cm})\end{array}$ & $\begin{array}{c}T z \\
(\mathrm{~cm})\end{array}$ & $\stackrel{s}{p p b}$ & $\begin{array}{c}s x \\
(m s)\end{array}$ & $\begin{array}{c}\sigma y \\
(m s)\end{array}$ & $\underset{(m s)}{a z}$ \\
\hline $\begin{array}{c}\text { WGS84 (G873) } \rightarrow \text { ITRF94 } \\
\text { Época 1997,0 }\end{array}$ & 9,6 & 6,0 & 4,4 & $-14,3$ & $-2,2$ & $-0,1$ & 1,1 \\
\hline $\begin{array}{c}\text { PZ-90 } \rightarrow \text { WGS84 (G873) } \\
\text { Época 1997,0 }\end{array}$ & $-108,0$ & $-27,0$ & $-90,0$ & $-120,0$ & 0,0 & 0,0 & $-160,0$ \\
\hline $\begin{array}{l}\text { ITRF8 } 8 \rightarrow \text { ITRF94 } \\
\text { Época 1988,0 }\end{array}$ & $-1,8$ & 0,0 & 9,2 & $-7,4$ & $-0,1$ & 0,0 & 0,0 \\
\hline $\begin{array}{l}\text { ITRF89 } \rightarrow \text { ITRF94 } \\
\text { Época } 1988,0\end{array}$ & $-2,3$ & $-3,6$ & 6,8 & $-4,3$ & 0,0 & 0,0 & 0,0 \\
\hline $\begin{array}{l}\text { ITRF90 } \rightarrow \text { ITRF94 } \\
\text { Época } 1988,0\end{array}$ & $-1,8$ & $-1,2$ & 3,0 & $-0,9$ & 0,0 & 0,0 & 0,0 \\
\hline $\begin{array}{l}\text { ITRF91 } \rightarrow \text { ITRF94 } \\
\text { Época } 1988,0\end{array}$ & $-2,0$ & $-1,6$ & 1,4 & $-0,6$ & 0,0 & 0,0 & 0,0 \\
\hline $\begin{array}{c}\text { ITRF92 } \rightarrow \text { ITRF9 } 4 \\
\text { Época } 1988,0\end{array}$ & $-0,8$ & $-0,2$ & 0,8 & 0,8 & 0,0 & 0,0 & 0,0 \\
\hline $\begin{array}{c}\text { ITRF93 } \rightarrow \text { ITRF94 } \\
\text { Época } 1988,0 \\
\end{array}$ & $-0,6$ & 0,5 & 1,5 & $-0,4$ & 0,39 & $-0,8$ & 0,96 \\
\hline $\begin{array}{l}\text { Variação dos } \\
\text { Partâmetros }\end{array}$ & $\begin{array}{c}\text { Tx } \\
\text { cm/ano }\end{array}$ & $\begin{array}{c}\dot{T y} \\
\mathrm{~cm} / \mathrm{ano}\end{array}$ & $\dot{\mathrm{Iz}}$ & $\left|\begin{array}{c}\dot{s} \\
\text { ppb/ano }\end{array}\right|$ & $\begin{array}{c}\dot{E x} \\
\text { ms/ano }\end{array}$ & $\begin{array}{c}\dot{y} \\
\text { ms/ano }\end{array}$ & $\begin{array}{c}\dot{z} \\
m \text { s/ano }\end{array}$ \\
\hline $\begin{array}{l}\text { ITRF93 } \rightarrow \text { ITRF94 } \\
\text { Época 1988,0 }\end{array}$ & 0,29 & $-0,04$ & $-0,08$ & 0,0 & 0,11 & 0,19 & $-0,05$ \\
\hline
\end{tabular}

Tabela 4 - Coordenadas e velocidades da estação ONSA, localizada na Suécia, referenciadas no ITRF92, ITRF93, ITRF94 e ITRF96.

Table 4 - Coordinates and velocity of the ONSA station, located in Sweden, in the ITRF92, ITRF93, ITRF94 and ITRF96.

\begin{tabular}{|c|c|c|c|c|c|c|c|}
\hline \multirow{2}{*}{ Referencial } & \multirow{2}{*}{ Época } & \multicolumn{3}{|c|}{ Coordenadas $(m)$} & \multicolumn{3}{|c|}{ Velocidades (m/ano) } \\
\hline & & $X$ & $Y$ & 2 & $V x$ & Vy & $V_{2}$ \\
\hline ITRF92 & 1988,0 & 3370658,810 & 711876,901 & 5349786,787 & $.0,0136$ & 0,0136 & 0,0118 \\
\hline ITRF93 & 1993,0 & 3370658,716 & 711876,978 & 5349786,830 & $.0,0152$ & 0,0133 & 0,0091 \\
\hline ITRF94 & 1993,0 & 3370658,732 & 711876,975 & 5349786,833 & $.0,0150$ & 0,0151 & 0,0061 \\
\hline ITRF96 & 1997,0 & 3370658,674 & 711877,032 & 5349786,866 & $.0,0143$ & 0,0147 & 0,0072 \\
\hline
\end{tabular}

aplicada nos casos em que entre os referenciais envolvidos, existem 7 parâmetros de transformação (3 translações, 3 rotações e 1 fator diferencial de escala) e mais 7 referentes as suas taxas de variação com relação ao tempo, totalizando 14 parâmetros, além das 3 componentes de velocidade $\left(v_{x}, v_{y}, v_{z}\right)$. Para exemplificar, pode-se tomar as coordenadas e o campo de velocidades da estação ONSA, referenciada no ITRF93, época 1993,0, transformando-a para o ITRF94 na época 1996,5. Para isso, aplica-se a Eq.(1) utilizando- 
se dos parâmetros da Tab.(3) e suas respectivas variações. No Exemplo (2) do Apêndice, apresenta-se uma situação em que essa versão é utilizada, onde são obtidas as coordenadas da estação ONSA, transformadas do ITRF93 para o ITRF94 na época 1996,5.

- Transformação com 7 parâmetros: é utilizada nos casos em que entre os referenciais envolvidos, as taxas de variação dos parâmetros não são disponíveis ou por serem pequenas podem ser desprezadas. Desta forma, nessa versão, aplica-se 3 translações, um fator diferencial de escala e 3 rotações, totalizando 7 parâmetros. Além destes, também faz parte deste tipo de transformação as componentes do vetor velocidade $\left(v_{x}, v_{y}, v_{z}\right)$. Este é o caso em que se enquadra uma transformação do ITRF92 para o ITRF94, uma vez que não há taxa de variação dos parâmetros entre estes referenciais. No Exemplo (3) do Apêndice mostra-se uma circunstância em que essa versão da transformação de Helmert generalizada se faz necessária. A estação utilizada é a ONSA, na Suécia, e a época é 1996,5.

-Transformação sem parâmetro (atualização): essa versão se aplica quando os parâmetros de transformação entre o referenciais envolvidos são muito pequenos, podendo ser desprezados. Nesse caso levase em consideração apenas a evolução temporal das estações ao aplicar as componentes do vetor velocidade $\left(v_{x}, v_{y}, v_{z}\right)$. Isto é o que ocorre, por exemplo, entre o ITRF94, ITRF96 e o ITRF97 (Soler, 1999). A Eq.(1), neste caso, é simplificada para a seguinte:

$$
\vec{X}_{B}=\vec{X}_{A}+\vec{V}_{A}\left(t-t_{0}\right) .
$$

Essa equação também pode ser aplicada para os casos em que se deseja determinar as coordenadas de uma estação em épocas diferentes, permanecendo no mesmo referencial (atualização). No Exemplo (4) do Apêndice é feita uma transformação onde a Eq.(18) é utilizada, tendo as coordenadas da estação ONSA, no ITRF96 época 1997,0, transformadas para o ITRF94, época 1996,5. Apesar de existir outras versões da transformação de Helmert generalizada, elas não serão tratadas aqui.

Note que nos 3 últimos exemplos contidos no Apêndice, os resultados estão em um mesmo referencial e mesma época, o que possibilita uma comparação entre os valores obtidos. Desta forma, aplicando a Eq.(18) nas coordenadas da estação ONSA referenciadas ao ITRF94, atualizando-as para a época 1996,5, obtêm-se os valores de referência. Tomando esses valores e comparando-os com os resultados

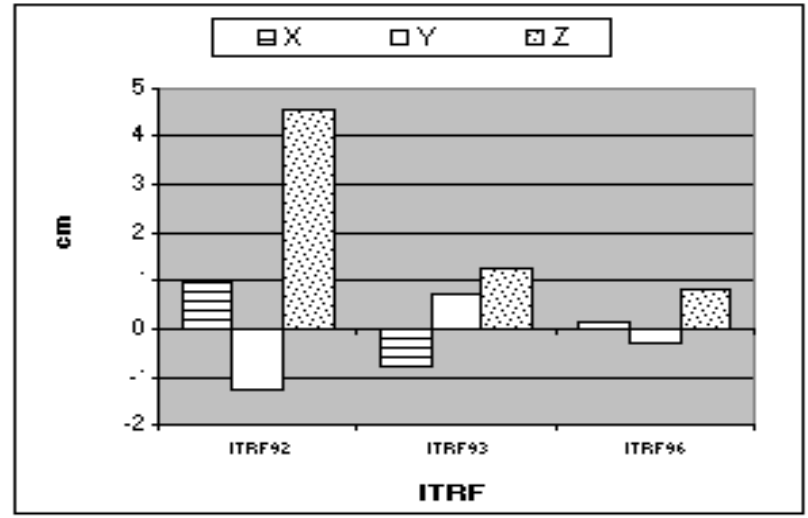

Figura 1 -Discrepâncias entre algumas realizações do ITRS na estação ONSA (Suécia), resultante da aplicação da transformação de Helmert generalizada para o ITRF94, época 1996,5.

Figure 1 - Discrepancies among some ITRS realizations of ONSA station (Sweden), resulting from the generalized Helmert transformation for ITRF94, epoch 1996, 5.

gerados nos demais exemplos, pode-se verificar discrepâncias entre eles. Na Fig.(1) são apresentadas as discrepâncias para as componentes $X, Y$ e $Z$. Esses resultados mostram que as realizações mais antigas, tal como esperado, apresentam piores resultados.

\section{COMENTÁRIOS FINAIS}

Os parâmetros utilizados ao aplicar a transformação de Helmert generalizada em trabalhos que se utilizam o posicionamento geodésico a partir de satélites artificiais, apesar de ser de pequena dimensão, são importantes para preservar a qualidade dos resultados. Isto é devido a melhoria considerável da acurácia dos resultados obtidos no posicionamento por satélites, que evoluíram nas últimas décadas e se tornaram sensíveis a pequenas variações. Anteriormente, tais parâmetros só tinham significado para resultados obtidos por técnicas mais avançadas, como o VLBI e o SLR, muito utilizadas em geodinâmica.

Os parâmetros de transformação destinados para as materializações do ITRS podem também ser aplicados para os casos dos referenciais de abrangência regionais, que estão associadas a um dos vários ITRFs, tendo atenção especial para a época em que foram materializados. Um exemplo desses referenciais é o SIRGAS (SIstema de Referência Geocêntrico para a América do Sul), que empregou o ITRF94 na época 1995,4 .

Os parâmetros de transformação apresentados na Tab.(3), no que concerne ao WGS84 e ao PZ90, devem ser aplicados com cautela, pois são pouco confiáveis. Com relação ao WGS84, isto se deve pela falta de 
significância estatística resultante do ajustamento que originou tais parâmetros (Malys et al., 1997). Quanto ao PZ90, o problema é a localização das estações que compunham a campanha da qual resultaram tais parâmetros, pois estas se localizam todas no território russo, não permitindo um alcance global (Bazlov et al., 1999). Inicialmente, os usuários que integram observações GPS e GLONASS para posicionamento geodésico, tiveram grande interesse nesses parâmetros. No entanto, ao invés de concentrar esforços objetivando refiná-los, decidiu-se referenciar as efemérides precisas dos satélites GLONASS também nas séries do ITRF, assim como o GPS (Allan, 1996). Com isso, passa-se a utilizar o campo de velocidades associado ao ITRF, sendo desnecessário conhecer o campo de velocidade associado ao WGS84 e ao PZ90, o que despenderia grandes esforços e resultaria em poucos benefícios.

Pode-se notar na Tab.(3) a ausência de algumas combinações entre as materializações mais antigas do ITRS, como por exemplo entre o ITRF90 e o ITRF91. Isto se justifica pela pouca aplicabilidade de tal transformação, uma vez que esses referenciais se restringem a poucas estações distribuídas mundialmente.

\section{CONCLUSÕES}

Apresentou-se nesse trabalho detalhes da transformação de Helmert generalizada, envolvendo sua dedução analítica e as versões que ela pode assumir. Tal transformação deve ser aplicada para os casos em que são empregados métodos de posicionamento geodésico que proporcionem alta precisão e estejam associados a referenciais bem definidos e materializados, como as várias séries do ITRF, o WGS84 e o PZ90. Quando se trata dos dois últimos, cuidados devem ser tomados quanto a confiabilidade dos parâmetros disponíveis, pois eles foram estimados com reduzido número de estações, com distribuição inadequada.

Com relação às discrepâncias geradas pela comparação dos resultados obtidos pelas transformações exemplificadas neste trabalho, podese afirmar que as componentes da estação ONSA, na época 1996,5, referenciadas ao ITRF93, ITRF94 e o ITRF96 são compatíveis ao nível de $1,3 \mathrm{~cm}$, e quando referenciada ao ITRF92 esse valor chega é de $5,5 \mathrm{~cm}$. A melhor compatibilidade das realizações mais recentes se deve, provavelmente, ao fato de haver uma serie maior de dados.

Avaliar a compatibilidade entre dois referenciais diferentes é importante pois fornece subsídios para prever a dimensão dos erros que podem ser cometidos nos trabalhos que os envolvem. Sabe-se que, com os resultados apresentados na Fig.(1), nada se pode afirmar quanto a compatibilidade entre os referenciais envolvidos, pois se trata de resultados de um caso isolado, mas aponta o caminho a ser tomado para que esse objetivo seja alcançado.

\section{APÊNDICE}

Neste Apêndice são apresentados exemplos numéricos que têm a função de aplicar alguns conceitos aqui tratados, facilitando a compreensão e eliminando dúvidas que possam surgir. $\mathrm{O}$ primeiro se refere a determinação do vetor velocidade de uma estação que não possui um campo dessa nela associado, enquanto os demais são similares e atentam por apresentar situações que necessitem das várias versões da transformação de Helmert generalizada mencionadas no texto.

\section{Exemplo 1:Determinação do vetor velocidade} para uma estação localizada sobre a placa litosférica América do Sul.

Problema: Suponha que alguém ao estudar a deformação da crosta na placa litosférica América do Sul, contasse com uma rede de estações distribuídas pela região de estudo. Conhecendo a posição dessas estações ao aplicar métodos de alta precisão e técnicas espaciais, deseja-se conhecer os valores aproximados das componentes da velocidade de uma delas, eleita como estação base.

Para resolver esse problema pode-se utilizar os valores das velocidades angulares da placa litosférica América do Sul, geradas pelo modelo NNR-NUVEL1A apresentadas na Tab.(2) em segundos por milhões de anos. Aplicando a Eq.(16) juntamente com as coordenadas cartesianas da estação base (tomada aqui, para efeito de exemplo, as coordenadas SIRGAS da estação UEPP da RBMC) em unidades metricas, obten-se a seguinte expressão :

$$
\begin{aligned}
\{v\}_{\text {Base }} \cong & \left.\cong \begin{array}{ccc}
0 & 0,87 & -1,515 \\
-0,87 & 0 & 1,038 \\
1,515 & -1,038 & 0
\end{array}\right] 10^{-9} \times \\
& \times\left[\begin{array}{ccc}
3.687 & .624,310 \\
-4.620 & .818 & , 571 \\
-2.386 & .880,407
\end{array}\right]=\left[\begin{array}{c}
-0,0004 \\
-0,0057 \\
0,0104
\end{array}\right],
\end{aligned}
$$

onde a matriz $[\Omega]$ da Eq.(16) é composta pelos valores da Tab.(2) transformados em radianos por ano e $\{v\}_{\text {Base }}$ 
é uma aproximação do vetor velocidade para a estação UEPP em metros por ano. O mesmo pode ser feito para as demais estações da citada rede, alterando apenas os valores das coordenadas na Eq.A1.

\section{Exemplo 2:Referenciando uma estação perten- cente ao ITRF93 no ITRF94.}

Problema: Suponha que ao desenvolver uma atividade Geodinâmica em 1995, um pesquisador determinou a posição de uma estação com alta precisão. A técnica utilizada foi o GPS na qual utilizou as efemérides precisas geradas pelo IGS. Como essas efemérides estavam referenciadas no ITRF93 (ver Tab.(1)), as coordenadas finais também ficaram associadas a esse referencial. Em meados de 1996 (época 1996,5) as coordenadas dessa estação foram novamente determinadas utilizando a mesma técnica. Como nesse período o IGS tinha suas efemérides no ITRF94, para que seja possível comparar os resultados adequadamente há a necessidade de referenciar as antigas coordenadas da estação no novo referencial.

Como essa situação é hipotética, não existindo realmente essa estação, a fim de ilustrar uma solução para o problema tomar-me-á a estação ONSA (localizada na Suécia), na época 1993,0 como sendo a estação em questão, cujas coordenadas e velocidades são apresentadas na Tab.(4). Para referenciar estas coordenadas no ITRF94, na época 1996,5, deve-se aplicar a Eq.(1) utilizando-se dos parâmetros da Tab.(3) e suas respectivas taxas de variação. Substituindo tais valores nesta equação chega-se na seguinte expressão:

$$
\begin{aligned}
& \{x\}_{\text {ITRF } 94 \text { epoca } 1996,5}^{\text {ONSA }}=\left[\begin{array}{r}
-0,006 \\
0,005 \\
0,015
\end{array}\right]+\left(1-0,4 \times 10^{-9}\right) \times \\
& \times\left[\begin{array}{ccc}
1 & 4,65 \times 10^{-9} & 3,87 \times 10^{-9} \\
-4,65 \times 10^{-9} & 1 & 1,89 \times 10^{-9} \\
-3,87 \times 10^{-9} & -1,89 \times 10^{-9} & 1
\end{array}\right] \times \\
& \times\left[\left[\begin{array}{r}
3.370 .658,716 \\
711.876,978 \\
5.349 .786,830
\end{array}\right]+\left[\begin{array}{r}
-0,0152 \\
0,0133 \\
0,0091
\end{array}\right](1996,5-1993,0)\right]+ \\
& +\left[\left[\begin{array}{r}
0,0029 \\
-0,0004 \\
-0,0008
\end{array}\right]+\left[\left(1-0,4 \times 10^{-9}\right) \times\right.\right. \\
& \left.\times\left[\begin{array}{ccc}
0 & -2,42 \times 10^{-10} & -9,21 \times 10^{-10} \\
2,42 \times 10^{-10} & 0 & 5,33 \times 10^{-10} \\
9,21 \times 10^{-10} & -5,33 \times 10^{-10} & 0
\end{array}\right]+[0]\right] \times \\
& \left.\times\left[\begin{array}{r}
3.370 .658,176 \\
711.876,978 \\
5.349 .786,830
\end{array}\right]\right](1.996,5-1.993,0)
\end{aligned}
$$

$$
\{x\}_{\text {ITRF } 94 \text { epoca } 1996,5}^{\text {ONSA }}=\left[\begin{array}{r}
3.370 .658,6718 \\
711.877,0351 \\
5.349 .786,8670
\end{array}\right] \text {, }
$$

Em um procedimento análogo chega-se na solução do problema levantado permitindo comparar os resultados obtidos nos diferentes posicionamentos hipoteticamente realizados.

\section{Exemplo 3:Referenciando uma estação perten- cente ao ITRF92 no ITRF94.}

Problema: Suponha que para uma determinada aplicação geodinâmica foi necessário conhecer com alta precisão as coordenadas de uma estação em junho de 1994. Para isso foi utilizado um método de posicionamento geodésico envolvendo a técnica GPS e as efemérides precisas do IGS, referenciadas no ITRF92. A partir dessa estação, tomada como base, através de um método de posicionamento relativo, foram implantadas outras estações, formando uma rede. Suponha também que depois de um período de interrupções dessa atividade geodinâmica, em meados de 1996 (época 1996,5) houve a necessidade de novas ocupações na estação base para adensamento dessa rede. Como as efemérides do IGS disponíveis nessa época estão referenciadas no ITRF94, necessita-se conhecer as coordenadas da estação base nesse outro referencial, na mesma época em que ocorreram as novas ocupações.

Para efeito ilustrativo, toma-se as coordenadas da estação ONSA, referenciada no ITRF92, na época 1988,0 . Tomando as coordenadas e as velocidades dessa estação nesse referencial (Tab.(4)) pode-se transforma-las para o ITRF94 época 1996,5 aplicandose os parâmetros de transformação apresentados na Tab.(3), utilizando-se da Eq.(1), em sua versão simplificada, pois parte desta se anula na ausência das taxas de variação dos parâmetros. Assim, ao aplicar os valores da Tab.(3) e Tab.(4) na Eq.(1), tem-se a seguinte expressão:

$$
\begin{aligned}
\{x\}_{\text {ITRF94(19965) }}^{\text {ONSA }} & =\left[\begin{array}{r}
-0,008 \\
-0,002 \\
0,008
\end{array}\right]+\left(1+0,8 \times 10^{-9}\right)\left[\begin{array}{lll}
1 & 0 & 0 \\
0 & 1 & 0 \\
0 & 0 & 1
\end{array}\right] x \\
& \left.\times\left[\begin{array}{r}
3.370 .658,810 \\
711.876,901 \\
5.349 .786787
\end{array}\right]+\left[\begin{array}{r}
-0,0136 \\
0,0136 \\
0,0118
\end{array}\right](19965-1988,0)\right]
\end{aligned}
$$

cujo resultado é:

de onde se obtém as seguintes coordenadas: 


$$
\{x\}_{\text {ITRF } 94}^{\text {ONSA }}=\left[\begin{array}{r}
3.370 .658,6891 \\
711.877,0152 \\
5.349 .786,8996
\end{array}\right] .
$$

Essas são as coordenadas mais adequadas para serem usadas em um posicionamento relativo, utilizando GPS e as efemérides precisas do IGS, tendo a estação ONSA como base na época 1996,5. O mesmo deveria ser feito para o caso da estação hipotética apresentada no problema acima.

Exemplo 4:Referenciando uma estação pertencente ao ITRF96 no ITRF94.

Problema: Seguindo o mesmo caminho dos exemplos anteriores, supõe-se conhecer as coordenadas de uma estação que tenham sido determinadas na época 1996,5 e estejam referenciadas no ITRF94. Suponha que no inicio de 1999, foi realizada uma nova determinação das coordenadas dessa estação, utilizando a técnica GPS e as efemérides produzidas pelo IGS. Como mostra a Tab.(1) essas novas coordenadas estão referenciadas no ITRF96, pois é o referencial das efemérides usadas. Deseja-se comparar as coordenadas resultantes dos diferentes posicionamentos na época e no referencial do primeiro deles, ou seja, no ITRF94 e na época 1996,5.

A solução é tomar as coordenadas resultantes do segundo posicionamento e aplicar uma transformação de Helmert generalizada utilizando a Eq.(1). Porém, os referenciais ITRF94 e ITRF96 são considerados idênticos, restando aplicar apenas o vetor velocidade, sendo simplificada para a Eq.(18).

Para ilustrar de forma numérica, nesta solução será utilizada as coordenadas e as velocidades da estação ONSA referenciadas no ITRF96, na época 1997,0, apresentadas na Tab.(4). Aplicando esses valores na Eq.(18) chega-se na seguinte equação:

$$
\begin{aligned}
\{x\}_{\text {ITRF } 94}^{\text {ONSA }} & =\left[\begin{array}{r}
3.370 .658,674 \\
711 \\
71877,032 \\
5.349 .786,866
\end{array}\right]+ \\
& +\left[\begin{array}{r}
-0,0143 \\
0,0147 \\
0,0072
\end{array}\right](1996,5-1997,0),
\end{aligned}
$$

de onde se obtém os seguintes resultados:

$$
\{x\}_{\text {ITRF } 94}^{\text {ONSA }}=\left[\begin{array}{r}
3.370 .658,6812 \\
711.877,0247 \\
5.349 .786,8624
\end{array}\right] .
$$

que são as coordenadas dessa estação no ITRF94 na época 1996,5. Esse exemplo mostra a possibilidade de retroceder no tempo as coordenadas da posição de uma estação, cuja precisão nos resultados dependem da qualidade do campo de velocidade empregado.

\section{AGRADECIMENTOS}

Este artigo contou com o suporte financeiro da FAPESP - Fundação de Amparo à Pesquisa no Estado de São Paulo, Processo No 99/04613-8.

\section{REFERÊNCIAS}

ALLAN, D. W. - 1996 - Harmonizing GPS and GLONASS. GPS World, 7(9): 51-54.

BAZLOV, E.A, GALAZIN, V.N., KAPLAM, B.L., MAKSIMOV, V.G. \& RODOZIN, V.P. 1999. GLONASS to GPS: A new Coordinate transformation. GPS World, 10(1): 54.

BOCK, Y., - 1998 - Reference System, In: Kleusber, A. \& Teunissen P. J. G., GPS for Geodesy, Berlin, Verlag, p 1-41.

BOUCHER, C. \& ALTAMINI, Z. - 1996 International Terrestrial Reference Frame, GPS World, 7(9): 71-74.

COSTA, S. M. A. - 1999 - Integração do Sistema Geodésico Brasileiro aos Sistemas de Referência Terrestres. Tese de Doutorado. Curso de PósGraduação em Ciências Geodésicas. UFPR, Curitiba, P.R. 156 pp.

GEMAEL, C. - 1994 - Introdução ao ajustamento de observações: aplicações geodésicas. Curitiba; Editora da UFPR, 319 pp.

GREGORIUS T. -1996- How it Works...GIPSY OASIS II, Departament of Geomaties University of Newcastle upon Tyne.

MAlYS, S., Slater, J. A., SMITH, R. W., KUNZ L.E. \& KEYON, S. C. - 1997 Refinements of the World Geodetic System 1994. Proc. $10^{\text {th }}$ Tech. Meeting of the Satellite Division of the Institute of Navigation, Kansas City, MO. The Institute of Navigation, Alexandria, VA, EUA. 841-850

MCCARTHY, D. D. - 1992 - IERS Standards, IERS Technical Note 13, Central Bureau of IERS Observatoire de Paris, França.

McCARTHY, D. D. - 1996 - IERS Technical Note 21. Central Bureau of IERS. Observatoire de Paris, França.

MONICO, J. F. G. \& SEGANTINE, P. C. L. - 
1996 - ITRF: Definição, Realizações e Aplicações. Cartografia e cadastro, Instituto Português, Lisboa, 5:31-36.

OLIVEIRA L. C. - 1998 - Realizações do Sistema Geodésico Brasileiro Associadas ao SAD 69: Uma proposta Metodológica de transformação. Tese de doutorado, Departamento de Transporte EPUSP, $197 \mathrm{pp}$.
SOLER, T. - 1998 - A compendium of transformation formulas useful in GPS work. Journal of Geodesy, 72 (7/8): 484-490.

SOLER, T. - 1999 - Transformações Rigorosas entre sistemas de Referência de coordenadas: Aplicação ao GPS (ITRF, WGS84) e GLONASS (PZ90) GeoConvergência, Março: 30-38.

\section{GENERALIZED HELMERT TRANFORMATION FOR HIGH PRECISION POSITIONING: THEORY AND EXAMPLES}

The applications of GPS (Global Positioning System) and GLONASS (Global Navigation Satellite System) techniques in geodynamics activities contribute to the studies of planet global changes. They allow the evaluation of geophysical phenomenon because of the high level of the precision provided in the determination of coordinates. It requires, however, a well-defined reference frame. The reference frames available for use with these techniques are the materializations of the WGS84 (World Geodetic System 1984), PZ90 (Parametry Zemli 1990) and ITRS (IERS -International Earth Rotation ServiceTerrestrial Reference System). It is frequently necessary to compare solutions materialized in different reference frames and different epochs. It requires applying the so-called generalized Helmert transformation, which considers the temporary evolution of the stations coordinates. Therefore, in this transformation are involved, besides the transformation parameters, their evolution with time. For the most recent ITRF, the velocity field is supplied together with the station coordinates list. Otherwise, a model should be used. It is also the case of WGS84 and PZ90. The NNR-NUVEL-1A is the more appropriate model and it is recommended by the IUGG (International Union of Geodesy and Geophysics). The transformation parameters required for use with the generalized Helmert transformation were estimated by applying adjustment methods using stations coordinates materialized at different reference frames. The transformation parameters that involve WGS84 and PZ90 reference frames should be applied with caution because they are less reliable, since fewer stations were used in their computation.

Due to the absence of transformation parameters among some reference frames, the generalized Helmert transformation can assume different versions. The principal ones are:

- 14 parameters transformation: used for the cases that there are 7 parameters ( 3 translations, 3 rotations and a factor scale) and 7 rates of variation of these parameters;

- 7 parameters transformation: used for the cases that there are the 7 transformation parameters; and

- Transformation without parameters: used for the cases that there are not transformation parameters, but only velocities.

The generalized Helmert transformation should be applied whenever high precision geodetic positioning is used, associated with a reference frame at different epochs. However, its application is relatively complex. In order to contribute with this theme, this paper presents the most relevant characteristics of this transformation, the available transformation parameters and the possible versions that it can assume, exemplifying them with applications.

\section{NOTE ABOUT THE AUTHORS}

\section{Luiz Fernando Sapucci}

Mestrando no Programa de Pós Graduação em Ciências Cartográficas da UNESP (Universidade Estadual Paulista), Campus de Presidente Prudente, São Paulo, Brasil, onde esta investigando a quantificação do vapor d'água atmosférico utilizando GPS em áreas do território brasileiro.
João Francisco Galera Monico

Professor Assistente no Departamento de Cartografia da UNESP (Universidade Estadual Paulista), Campus de Presidente Prudente, São Paulo, Brasil, onde leciona e pesquisa desde 1982. Ele recebeu o titulo de $\mathrm{PhD}$ em Engenharia de levantamentos e Geodésia Espacial da The University of Nottingham, Reino Unido em 1995. Ele está envolvido com posicionamento de alta precisão e aplicações GPS não convencionais. 